\title{
Design and Evaluation of Teacher Assistance Tools for Exploratory Learning Environments
}

\author{
Manolis Mavrikis \\ London Knowledge Lab \\ UCL Institute of Education \\ m.mavrikis@ucl.ac.uk
}

\author{
Sergio Gutierrez-Santos \\ London Knowledge Lab \\ Birkbeck, Univ. of London \\ sergut@dcs.bbk.ac.uk
}

\author{
Alex Poulovassilis \\ London Knowledge Lab \\ Birkbeck, Univ. of London \\ ap@dcs.bbk.ac.uk
}

\begin{abstract}
We present our approach to designing and evaluating tools that can assist teachers in classroom settings where students are using Exploratory Learning Environments (ELEs), using as our case study the MiGen system, which targets 1114 year old students' learning of algebra. We discuss the challenging role of teachers in exploratory learning settings and motivate the need for visualisation and notification tools that can assist teachers in focusing their attention across the whole class and inform their interventions. We present the design and evaluation approach followed during the development of MiGen's Teacher Assistance tools, drawing parallels with the recently proposed LATUX workflow but also discussing how we go beyond this to include a large number of teacher participants in our evaluation activities, so as to gain the benefit of different view points. We discuss the results of the evaluations, which show that participants appreciated the capabilities of the tools and were mostly able to use them quickly and accurately.
\end{abstract}

\section{Categories and Subject Descriptors}

J.1 [Administrative Data Processing]: Education; K.3 [Computers and Education]: Computer Uses in Education - Computer assisted instruction, computer managed

\section{Keywords}

teacher assistance tools, exploratory learning

\section{INTRODUCTION}

Design and evaluation of learning analytics tools targeted at the teacher is not an easy task and, as discussed in [9], requires the adoption of interdisciplinary techniques and methods. We present here our approach to designing and evaluating tools that can assist teachers in a classroom where students are using Exploratory Learning Environments (ELEs). Examples of ELEs include simulators, microworlds, virtual

Permission to make digital or hard copies of all or part of this work for personal or classroom use is granted without fee provided that copies are not made or distributed for profit or commercial advantage and that copies bear this notice and the full citation on the first page. Copyrights for components of this work owned by others than the author(s) must be honored. Abstracting with credit is permitted. To copy otherwise, or republish, to post on servers or to redistribute to lists, requires prior specific permission and/or a fee. Request permissions from permissions@acm.org.

LAK '16, April 25 - 29, 2016, Edinburgh, United Kingdom

(C) 2016 Copyright held by the owner/author(s). Publication rights licensed to ACM. ISBN 978-1-4503-4190-5/16/04 . .\$15.00

DOI: http://dx.doi.org/10.1145/2883851.2883909 labs, and educational games, all of which give considerable freedom to students to learn in a variety of different ways.

The role of teachers in such an exploratory learning setting is that of a 'facilitator', or 'orchestrator' $[11,3]$. This role would be relatively easy in one-to-one student-tutor interaction, but scaling it up to the number of students present in a typical classroom poses several challenges, further compounded by the use of technology [3]. Given the open-ended nature of the tasks that the students are working on, teachers can only be aware of what a small number of students are doing at any one time as they walk around the classroom. The computer screens of students who are not in their immediate vicinity are typically not visible to the teacher, and it is therefore hard to know which students are making progress, which are off-task, and which are in difficulty and in need of additional support.

Our case study here is the MiGen system (see migen.org), which includes an intelligent microworld called eXpresser, designed to support 11-14 year old students' development of algebraic ways of thinking. As part of the MiGen system, we have designed a suite of visualisation and notification tools, which we refer to as the Teacher Assistance (TA) tools, whose aim is to assist teachers in focusing their attention across the whole class as students are working with eXpresser, and to inform teachers' own interventions.

Earlier work has described the architectural design and implementation of the TA tools, focusing specifically on one tool, the Student Tracking (ST) tool [6]. In contrast, the present paper presents the design and evaluation approach we followed for the whole suite of TA tools. Our evaluation approach resembles, retrospectively, the LATUX (Learning Awareness Tools User eXperience) workflow recently put forward for designing and deploying awareness tools in the classroom [9]. LATUX proposes an iterative process of five phases common in software engineering and user experience approaches - Problem Definition, Low and Higher-fidelity Prototyping, Pilot studies and Validation in-the-wild.

In this paper we go beyond the Validation in-the-wild stage. We discuss our efforts to ensure the quality of our TA tools and of the user experience, as well as identifying metrics that provide us with indicators of the usability of the TA tools and of our success in addressing the requirements emerging from earlier stages. Extending the LATUX approach, we discuss our efforts to include a large number of teacher participants in our summative evaluation activities, so as to gain the benefit of different view points, while recognising that this can be a costly and time-consuming effort. 
The structure of the paper is as follows. Section 1 has introduced and motivated the research. Section 2 gives an overview of the context and functionalities of the MiGen system, and of related work in ELEs and support for the teacher. Section 3 discusses the methodology we have adopted in designing, developing and evaluating the TA tools. We also discuss teachers' requirements from these tools, in the form of a set of usage scenarios. Section 4 describes the TA tools themselves, as well as a time-stop functionality that we developed specially for facilitating user evaluation with large numbers of teacher participants. Sections 5 presents our evaluation. Section 6 discusses the results and gives our concluding remarks and directions for further research.

\section{BACKGROUND AND RELATED WORK}

\subsection{The MiGen system and eXpresser}

The MiGen project has designed and developed an intelligent, exploratory environment to support 11 to 14 -yearold students learning of algebraic generalisation. Using the eXpresser mathematical microworld, students are asked to construct two-dimensional tiled models and associated algebraic rules. In order to build a model, students need to create 'building blocks' out of unit-square coloured tiles, based on their perception of the model's structure, and to repeat each building block so as to create a 'pattern' that forms part of their overall model. The algebraic rules they construct relate to the number of tiles of each colour required to paint each pattern and their model overall.

The eXpresser has an 'animation' facility which allows students to explore the generality of their models and rules. This applies automatically different random values to the variables used by the student and displays the resulting instances of the model in a separate pane of their screen. Tasks are designed so as to contextualise students' interaction with the eXpresser, and include a set of goals for students to achieve, e.g. 'check that your model is always coloured'.

ELEs such as MiGen's eXpresser have the potential to support students' exploration while at the same time fostering progressive building of knowledge. For example, eXpresser has been specifically designed to support students with some well-known and well-researched challenges on learning algebra. In particular, eXpresser has certain 'epistemic affordances' aimed at enhancing students' understanding of algebraic generalisation [12].

As students are undertaking the current task that they have been set using the eXpresser, a series of indicators are automatically detected by the system and stored in a database hosted on the MiGen Server (see [6] for details). The indicators that are meaningful and useful for teachers in their role in the classroom have been identified through an iterative process undertaken collaboratively with our group of teacher collaborators (see Section 3). There are two categories of indicators: task independent (TI) and task dependent (TD). TI indicators refer to aspects of the student's interaction that are related to the eXpresser microworld itself and do not depend on the specific task the student is working on, e.g. 'placed a tile on the canvas', 'created a pattern'. In contrast, the detection of TD indicators uses knowledge of the task that the student is working on and requires intelligent reasoning by the system, e.g. 'student has made a plausible building block for this task', 'student has coloured their pattern generally'.
The TA Tools (see Section 4) receive real-time notifications from the MiGen Server relating to occurrences of TI and TD indicators for each student, and each TA tool visualises a selection of this information to the teacher.

\subsection{Related Work}

To our knowledge, MiGen's TA tools represent the first work targeted at providing teachers with information about students' progress and state during exploratory learning activities in the classroom, notifying them of students' attainment of key indicators, and aiming to inform the teacher's own interventions in the class. This novelty of our TA tools has presented a number of methodological challenges, which we discuss in Section 3. In the past few years, several similar initiatives have appeared, including a recent approach that built on the work described here and that aimed to help teachers working with the Metafora platform and its associated tools, which target science and mathematics education [4]. Most related work investigates students' collaborative interactions while collaborating at interactive tabletops [9] or other multi-touch technology(e.g. [14]). The trend towards teacher support is recently growing also in the learning analytics community $[5,15]$ and there is high synergetic potential between that work and the work reported here. Other earlier initiatives include using Web log data generated by course management systems (e.g. WebCT) to help instructors become aware of students' activities in distance learning classes [13]; or class-wide collaborative activities supported by hand-held devices [2]. However, with the notable exception of a few works, e.g. [1] that visualises students' inferred plans in an ELE for chemistry, none of this work focuses on exploratory learning activities.

\section{METHODOLOGY}

In our experience over many years of working with maths teachers in participatory design and research projects, teachers are not accustomed to having access to tools such as MiGen's TA tools when teaching. Their typical instinct is to walk around the classroom in order to monitor how individual students are progressing and to help them. This makes it difficult, from the outset, to elicit from teachers a set of requirements for tools to support them. Instead, it was necessary to adopt an iterative participatory methodology, comprising successive phases of prototyping, requirements elicitation, incremental development, and evaluation.

Our earlier work, described in [6], presented the methods and outcomes of a first requirements elicitation and design phase for the TA tools. During this first phase, mockups and prototypes of possible visualisations for the TA tools were developed and discussed in several meetings of the project's Teacher Advisory group. As reported in [6], this advisory group comprised around 20 maths teachers and mathematics educators from a broad spectrum of secondary schools in the greater London area, who attended regular project team meetings and gave their input throughout the project. However, the time that these teachers had available to actually use prototypes of the tools in their classrooms was limited, and so collaboration with a core group of four teachers who trialled the tools played a prominent role in subsequent phases. Two teachers in particular piloted successive limited prototypes of the tools in classroom sessions.

Given these challenges, to help us get even to a stage of meaningful problem identification and elicit teachers' re- 
quirements for the TA tools, we had to short-circuit the LATUX workflow and to perform a rapid iteration of the whole cycle with prototype tools that were a little more than low-fidely prototypes - they were not paper-based but fully functional (albeit limited in their scope). We referred to this approach as 'iterative context engineering' (see [10]) because we 'engineered' settings that gave teacher participants the opportunity to experience first-hand what it would mean to have access to such TA tools and, therefore, to offer deeper insights in susbequent one-to-one interviews. Based on these interviews, but also through feedback from our general teacher advisory group, a set of Usage Scenarios for the whole suite of TA tools were identified and the tools were refined accordingly. These Usage Scenarios are as follows:

1. Finding out which students need the teacher's immediate help.

2. Finding out which students are progressing satisfactorily towards completing the task and which students may be in difficulty.

3. Finding out which students are currently disengaged from the task.

4. Identifying common conceptual and procedural difficulties that students are facing in order to provide more explanation to the class as a whole.

5. Finding out which students have finished the task.

6. Finding out which students have achieved which task goals.

7. Providing appropriate support and guidance to individual students: (i) during the lesson, and (ii) after the lesson.

8. Reflecting on the achievements of the class and planning the next lesson.

The establishment of these usage scenarios informed subsequent development iterations and allowed us to orient the evaluation activities around them. In particular, we followed a process that resembles Stages 3 (higher-fidelity prototyping), 4 (pilot) and 5 (validation-in-the-wild) of the LATUX workflow. Having held several focus groups, and undertaken small pilots, our challenge was to get input and evaluate the potential of the TA tools against these usage scenarios by a large number of teachers in a cost-effective way. Given the constraints of funded research and of the limited time that schools have for engaging in a research project, it was not possible to undertake a full cycle of using the whole MiGen system and the TA tools in the classroom over several hours. We therefore needed a methodology that would allow us to evaluate the tools on our premises with several people at a time but in a way that would provide the participants with a realistic experience of using the tools in the classroom. We present this approach and its results in Section 5.

\section{THE TEACHER ASSISTANCE TOOLS}

MiGen's suite of TA tools consists of the Student Tracking (ST), Classroom Dynamics (CD), and Goal Achievements (GA) tools. A fourth tool - the Grouping Tool [8] — is not discussed here as it does not relate directly to monitoring students' activities and progress.

The ST tool is the most detailed TA tool, and the one developed first chronologically. It monitors the occurrence of task-independent and task-dependent indicators generated by each student as they interact with the eXpresser. Coloured indicators are displayed in chronological order in a top-down 'timeline' for each student. Green/red indicators show productive/problematic interaction with respect to the task set. Yellow indicators show that the student's interaction may be positive or negative depending on context. Blue indicators relate to feedback provided to the student by the system. The teacher can select which indicators should be shown and which hidden, depending on her current needs. For reasons of space, we refer the reader to [6] for further description and screenshots of the ST tool.

The CD tool gives the teacher an at-a-glance overview of which students are currently engaged with the task and which may be in difficulty and in need of help from the teacher (see Figure 1, left-hand side). It represents each student present in the classroom by a colour-coded circle, containing the student's initials. Hovering over a circle with the cursor displays the student's full name. Clicking on a circle shows the student's current model and rule (see Figure 1 , right-hand side).

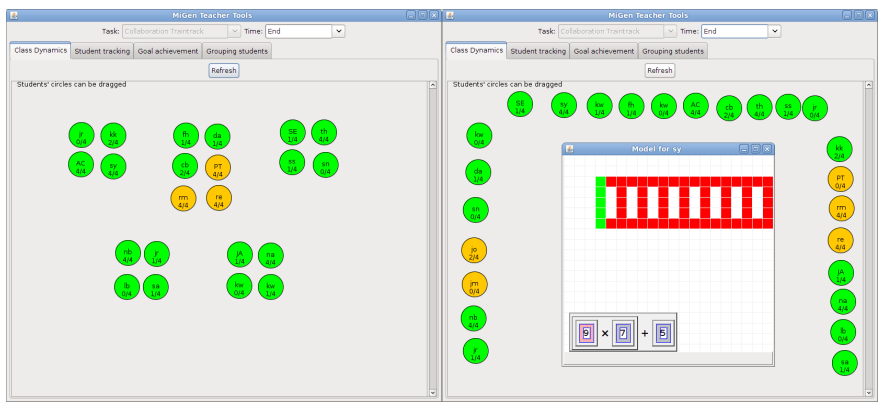

Figure 1: Class Dynamics tool. On the left, a classroom with the students sitting at tables. On the right, a U-shaped classroom; in this case, the teacher has clicked on one of the students to see their model and rule. The colour of a student's circle reflects the student's current activity status as perceived by the system. Green circles show students working productively on the task set. Amber circles show students who have not interacted with eXpresser for some time (by default, five minutes). Red circles show students who may benefit from immediate help.

In the CD tool, the circles representing the students can be dragged and moved around on the canvas. This enables teachers to set up the display so that the position of the circles matches the students' spatial positions in the classroom. This helps the teacher to match the information being displayed in the CD tool with her own observations. It also helps the teacher to identify situations that may be locationdependent. For example, if several students seated at the same table show as Amber this may indicate that they are distracting each other.

The GA tool shows a tabular display of students and task goals (see Figure 2). Each row of the table shows the progress of one student (identified by their initials) in completing the task goals. Each column shows the completion status of one task goal across all students. Hovering over a cell with the cursor displays a full description of the goal, the name of the student, and the achievement status of that goal for that student.

A cross-tool functionality we term 'time-stop' is supported 


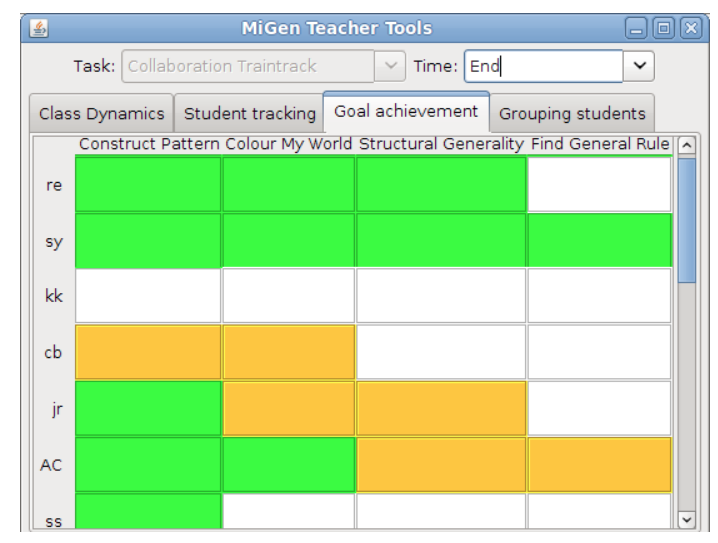

Figure 2: Goal Achievement tool. Green and white cells show whether a goal has been achieved or not. Amber shows that the goal has been achieved earlier but the student's current construction is not meeting the goal criteria.

by all the TA tools. It allows the user to select a specific point in time, $t$, with respect to which the ST, CD and GA visualisations are generated. The tools ignore all indicator occurrences after that time point, allowing analysis of the classroom situation at that particular time. If the time point $t$ selected is in the future, or if no time point is selected, the tools show the current situation by default.

\section{EVALUATION}

As mentioned in Section 3, early stages of our design approach established several usage scenarios through fully functional but limited prototypes piloted with a small group of teachers. Having undertaken these pilots, and having tested the TA tools thoroughly, we engaged first in a 'higher fidelity prototyping' and focus groups with several teachers. After taking into account participants' feedback, we then engaged in a small pilot and subsequently a 'validation inthe-wild' classroom-based trial. This trial involved one of our teacher collaborators at her school and aimed at allowing her to compare the difference in the teacher's experience compared to a lesson in which she did have use of the TA tools. We present the results in [7].

In order to get input from several teachers and to further evaluate the tools we held a 2-hour session with a cohort of 11 Maths teachers on the PGCE programme at the Institute of Education. Each participant had an installation of the MiGen system running on their computer. In the first half of the session, they were introduced to the MiGen system as a whole, the eXpresser, and the TA tools. They were then asked to work through several construction examples using eXpresser so as to gain familiarity with how students would use it in a lesson and the kinds of feedback the system would give to students. In the second half of the session, each of the TA tools was introduced to the participants, using real data drawn from a previous classroom pilot. Participants were asked to use the TA tools and the time-stop functionality to answer the following questions relating to Usage Scenarios 1-6 at different time points in the lesson, simulating the use of the tools in an actual classroom.

- Q1. The session started 10 minutes ago (10 minutes into the lesson). If you chose a student to help immediately, which student(s) would you choose and why?
- Q2. Based on your experience and previous sessions, you would have expected by now (10 min. on) that students have achieved at least two goals. With a quick glance of the tools would you say that the class is going according to plan or would you intervene and why?

- Q3. We are at 30 minutes on. Based on your experience and previous sessions, you expected that students would have finished by now so that you can progress on the next task. With a quick glance of the tools do you think that the class is at that stage and why?

- Q4. Sometimes students are off-task (e.g. play games). At 30 minutes on, find two students that are disengaged/distracted.

- Q5. We are at 30 minutes. Some students need help and you are trying to identify others who have finished and can help them. Can you give two examples of students who have finished?

Participants were also asked how long they thought it would take them to answer these questions in an actual lesson, our aim being not only to determine if participants were able to use the TA tools to answer the questions correctly, but also how they perceived the amount of time that it would take them to answer the questions in a classroom situation.

All participants provided correct answers to all questions without any assistance from the research team. The graph in Figure 3 summarises their responses relating to the perceived length of time required to answer each question. We see that for all the questions no participant responded a long or a lot of time. The questions regarded as requiring the least time to answer were Questions 1, 4 and 5, most probably because they pertain to individual students and could be answered by consulting just one tool (the CD tool). Questions 2 and 3 may have appeared to participants as needing more time to answer because they refer to the classroom as a whole and because, in order to answer them, participants may have consulted the GA too, and also the ST tool in Question 2 for a more detailed view of how students are progressing with their constructions.

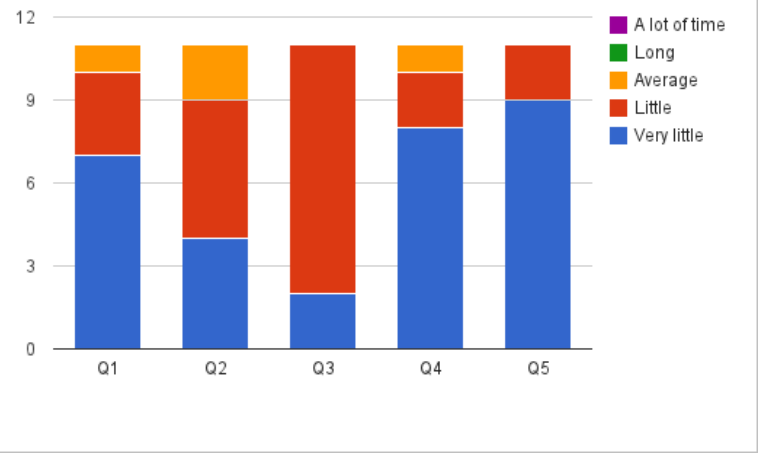

Figure 3: Participants' perceived time to answer evaluation questions

On the whole, we consider these responses as being encouraging, particularly as no question was perceived as requiring a long or a lot of time, which was a key aim in the design of the TA tools. 


\section{DISCUSSION AND CONCLUSION}

In this paper, we have described a suite of Teacher Assistance (TA) tools that target an exploratory learning setting, and their summative evaluation. Our overall approach resembles and validates, retrospectively, the LATUX workflow but also elaborates on a possible extension of the validationin-wild stage. Our particular methodological contribution was to extend the number of teacher participants and the type of data that can be gathered to evaluate tools such as our TA tools. The provision of time-stop functionality across all of the TA tools allowed us to use real interaction data from earlier classroom trials and to present these data to several participants via the TA tools 'frozen' at particular moments in time, simulating in this way the experience of using the tools in an actual classroom. This approach helped us conduct evaluations with a far greater number of teachers than those who would ever be able to participate in classroom trials either due to their constraints or to limited resources on our behalf. As such we consider it a type of 'poor man's evaluation at scale'.

The evaluation results are encouraging. The results show that teachers exposed to the TA tools understand the tools' capabilities and are able to use them effectively in answering most of the usage-scenario based questions. Answering most of the evaluation questions was relatively speedy, which achieves one of our objectives and is a key requirement for using the TA tools in a real classroom.

The TA tools presented in this paper are general in their design and similar tools could be used to monitor the activities of students interacting with other exploratory learning environments, provided that the environment detects appropriate interaction indicators. This would need to include, as a minimum, indicators relating to students' current activity status, waiting for help from the teacher, and goal achievements status: these are the indicators that drive the CD and GA tool visualisations which we have found that, in practice, teachers consult most often during a lesson.

Having received more feedback on the TA tools from teachers since they were made more widely available, it is evident that tools such as these allow teachers to use ELEs in the classroom in ways that were not possible before. Visualisation and notification tools that are developed specifically to support the teacher in the classroom are better able to provide a sense of awareness than other general-purpose screen monitoring tools. Moreover, with the increased emphasis on evidence-based teaching, such TA tools empower teachers to provide evidence of learning, even in a context that is less subject to formal assessment, and to engage in their own inquiry into more conceptual student learning. Our future work involves investigating how the TA tools could be adapted to support teachers in even more complex learning scenarios, such as blended learning, and in their own professional development as teachers.

\section{REFERENCES}

[1] O. Amir and K. Gal. Plan recognition and visualization in exploratory learning environments. ACM Transactions on Interactive Intelligent Systems, 3(3), 2013. DOI: $10.1145 / 2533670.2533674$.

[2] C. Cortez, M. Nussbaum, G. Woywood, and R. Aravena. Learning to collaborate by collaborating: a face-to-face collaborative activity for measuring and learning basics about teamwork. Journal of Computer Assisted Learning, 25(2):126-142, Apr. 2009.

[3] P. Dillenbourg. Design for classroom orchestration. Computers 85 Education, 69:485 - 492, 2013.

[4] T. Dragon, M. Mavrikis, B. McLaren, A. Harrer, C. Kynigos, R. Wegerif, and Y. Yang. Metafora: A web-based platform for learning to learn together in science and mathematics. IEEE TLT, 6(3), 2013.

[5] R. C. Garcia, A. Pardo, C. D. Kloos, K. Niemann, M. Scheffel, and M. Wolpers. Peeking into the black box: visualising learning activities. International Journal on Technology Enhanced Learning, 4(1/2):99-120, 2012.

[6] S. Gutierrez-Santos, E. Geraniou, D. Pearce-Lazard, and A. Poulovassilis. Design of Teacher Assistance Tools in an Exploratory Learning Environment for Algebraic Generalization. IEEE Transactions on Learning Technologies, 5(4):366-376, 2012.

[7] S. Gutierrez-Santos, M. Mavrikis, G. E., and A. Poulovassilis. Usage scenarios and evaluation of teacher assistance tools for exploratory learning environments (under review). Technical report, 2012. Available at http://www.dcs.bbk.ac.uk/research/ techreps/2012/bbkcs-12-02.pdf.

[8] S. Gutierrez-Santos, M. Mavrikis, E. Geraniou, and A. Poulovassilis. Similarity-based grouping to support teachers on collaborative activities in exploratory learning environments. IEEE Transactions on Emerging Topics in Computing, in press.

[9] R. Martinez-Maldonado, A. Pardo, N. Mirriahi, K. Yacef, J. Kay, and A. Clayphan. The LATUX workflow: Designing and deploying awareness tools in technology-enabled learning settings. In Proceedings of the Fifth International Conference on Learning Analytics And Knowledge, pages 1-10, 2015.

[10] M. Mavrikis et al. Iterative context engineering to inform the design of intelligent exploratory learning environments for the classroom. In R. Luckin et al., editors, Handbook of Design in Educational Technology, pages 80-92. Routledge, 2013.

[11] M. Mavrikis, S. Gutierrez-Santos, E. Geraniou, and R. Noss. Design requirements, student perception indicators and validation metrics for intelligent exploratory learning environments. Personal and Ubiquitous Computing, 17:1605-1620, 2013. DOI: http://dx.doi.org/10.1007/s00779-012-0524-3.

[12] M. Mavrikis, R. Noss, C. Hoyles, and E. Geraniou. Sowing the seeds of algebraic generalization: designing epistemic affordances for an intelligent microworld. Journal of Computer Assisted Learning, 2012.

[13] R. Mazza and V. Dimitrova. CourseVis: A graphical student monitoring tool for supporting instructors in web-based distance courses. International Journal of Man-Machine Studies, 65(2):125-139, 2007.

[14] E. Mercier, G. Vourloumi, and S. Higgins. Student interactions and the development of ideas in multi-touch and paper-based collaborative mathematical problem solving. BJET, 2015.

[15] V. A. R. Zaldivar, A. Pardo, D. Burgos, and C. D. Kloos. Monitoring student progress using virtual appliances: A case study. Computers $\&$ Education, 58(4):1058-1067, 2012. 